\title{
A Comparative Study of Percutaneous Catheter Drainage Versus Percutaneous Needle Aspiration In The Treatment of Medium to Large Sized Liver Abscess
}

\author{
Dr.Sudeep Nagori, Dr.Paresh Sodhiya, Dr.R.K.Mathur, Dr.Ravindra Chaudhary
}

\begin{abstract}
Objective: To compare the effectiveness of percutaneous catheter drainage (PCD) and percutaneous needle aspiration (PNA) in the management of medium to large (>5 cm diameter) sized liver abscesses.

Method: Seventy-two patients with liver abscess (amoebic 48, pyogenic 24) were randomly allocated to PCD or PNA (36 each), which were done within 24 hours of admission. Both groups received parenteral antibiotics for atleast 10 days. PNA was repeated every third day if the cavity size had not declined to $50 \%$ of the original for up to three times.
\end{abstract}

Persistence of cavity or of clinical symptoms was considered failure of treatment. Duration to attain clinical relief, duration of hospital stay, complications, treatment failure, and deaths were recorded.

Results PNA was successful in 31 of $36(86 \%$ ) patients (one aspiration in 10, two in 18, and three in 3 patients), whereas PCD was successful in 35 (97\%) patients $(p=n s)$. Duration to attain clinical relief (10.2 [2.0] vs. 8.1 [2.7] days; $p=0.02)$ and parenteral antibiotics needed (15.5 [1.1] vs. 10.9 [2.7] days; $p=0.04)$ were significantly lower in PCD group. Duration of hospital stay was similar in the two groups. One patient with PNA had a subcapsular hematoma and one with PCD had continuous bile leakage which stopped spontaneously. One patient in PCD group died.

Conclusion: PCD is a better treatment option than PNA for the management of medium to large liver abscesses of size $>5 \mathrm{~cm}$ in terms of duration to attain clinical relief and duration for which parenteral antibiotics are needed.

Keywords: Amebic liver abscess, Entamoeba histolytica, Pyogenic abscess of liver, Percutaneous catheter drainage.. Percutaneous needle aspiration

\section{Introduction}

Amebic liver abscess (ALA) and pyogenic liver abscess (PLA) are common clinical problems in India and other tropical countries. ${ }^{1,2}$ In these areas, patients often present late when the abscess becomes large. Currently, these patients are treated with antibiotics along with percutaneous needle aspiration (PNA) or percutaneous catheter drainage (PCD), with surgical drainage being used only in patients who fail to respond to such treatment. ${ }^{3-6}$ Though both PNA and PCD have been shown to be effective and safe, ${ }^{7-9}$ data comparing these procedures are limited. We could find only three previous randomized studies comparing these two treatment options. These included liver abscesses of all sizes and two studies included only patients with PLA. ${ }^{7-}$ 9 .We therefore conducted a study to compare the effectiveness of PCD and PNA in the management of medium to large sized liver abscess of size $5 \mathrm{~cm}$ or more.

\section{Methods}

Patients with ALA or PLA exceeding $5 \mathrm{~cm}$ in the largest diameter, admitted to our institution Maharaja Yashwantrao Hospital, Indore in last 2 consecutive years 2014 \& 2015 were enrolled. Diagnosis of liver abscess was made by ultrasonography (USG). Patients with coexisting malignant biliary disease were excluded. A local scintific committee approved the study. Of the 80 consecutive patients initially enrolled, five were excluded because of abscess rupture before intervention, and one each because of coexisting carcinoma gall bladder, periampullary carcinoma and necrotizing amoebic colitis with peritonitis. All the patients gave written informed consent. Immediately after the diagnosis of liver abscess, empirical intravenous antibiotic therapy with ceftriaxone $1 \mathrm{~g}$, gentamicin $1 \mathrm{mg} / \mathrm{kg}$ and metronidazole $7.5 \mathrm{mg} / \mathrm{kg}$ was administered three times a day .A pus sample was obtained for microscopy, culture and antibiotic sensitivity, and hemagglutination (IHA) test for amoeba. Diagnosis of PLA or ALA was based on US and/or CT findings, and pus characteristics. If 
culture was positive, anti-microbial therapy was modified based on the results of sensitivity testing. Intravenous antibiotics were continued for at least 10 days and until fever had subsided for at least 48 hours; this was followed by appropriate oral antibiotics for the next 4 weeks.

\section{Needle aspiration}

Pus was aspirated under US guidance using a $16 \mathrm{G}$ disposable trocar needle and syringe. In multiloculated abscesses, the needle tip was inserted into various loculi for complete pus removal. Review USG was done every third day, and the size of the residual cavity was noted. Aspiration was repeated if the abscess cavity did not show a 50\% reduction in size, irrespective of clinical response. Failure of abscess size to decline below $50 \%$ of the original or no significant clinical improvement after third aspiration was taken as failure of aspiration.

\section{Catheter drainage}

A trocar with a 14 Fr multi-sidehole pigtail catheter was inserted into the abscess cavity under local anesthesia and US guidance. The contents of the abscess were aspirated and the catheter was fixed to the skin and connected to a bag. The first review US was done when drainage over the last 24 hours had declined to $<10$ $\mathrm{mL}$. If the abscess had resolved, the catheter was removed. If residual cavity was present, the catheter was flushed with saline and aspirated till no material was aspirated. Any loculations of residual cavity were treated with catheter manipulation under local anesthesia. Further review USG were done every third day and the catheter was removed if the catheter drainage had remained minimal. Otherwise, the catheter was left until catheter drainage had stopped. USG was repeated until the cavity had either decreased by $50 \%$ or more of its original size or been static with clinical recovery.

\section{Follow-up}

Patients were assessed daily for clinical improvement and for abscess size using USG. Intervention was considered successful, if there was complete clinical recovery and disappearance of abscess cavity. Duration to attain clinical recovery (relief of pain and fever), duration of antibiotic use, duration of hospital stay, complications, failure of intervention, and death were recorded. After discharge, the patients were followed clinically and by USG, in the outpatient clinic biweekly for the first 2 months and then monthly for the next 4 months.

\section{Results}

Of the 72 patients, 36 were randomized to PCD and 36 to PNA. The two groups had similar patient characteristics and underlying diseases (Table 1). Patients in the PNA group more often had jaundice (8/36 vs. $3 / 36$ in PCD, $\mathrm{p}=0.189)$ and anorexia/malaise (32/36 vs. 22/36 in PCD, $\mathrm{p}=0.003)$.

The baseline clinical features and biochemical parameters of all the patients analyzed in the two groups were similar (Table 1). The number, location, and nature (pyogenic or amebic) of abscesses were also similar. The aspiration group had a larger number of patients with left liver lobe abscesses $(7 / 36 \mathrm{vs} .4 / 36, \mathrm{p}=0.514)$ and multiple abscesses (8/36 vs. 5/36, p=0.354). Of the 72 cases, $48(67 \%)$ had amebic abscess and 24 had pyogenic liver abscesses (PLA). Among cases with PLA, pus culture was positive in 13 cases; it showed Escherichia coli in nine cases, of whom five were also positive for Klebsiella spp. Two specimens grew Pseudomonas, and one each grew Staphylococcus aureus and Bacillis fragilis. Gall bladder and CBD calculi was the most common coexisting pathology being present in $23(32 \%)$ cases, followed by cholangitis $(n=11)$ and diabetes mellitus $(n=8)$. Sixty-six percent (48/72) of patients had leukocytosis (TLC $>11,000 / \mathrm{mm} 3$ ). Prothrombin time-INR was raised above 2 in 22 patients (30\%), and serum bilirubin was $>2.0$ in $25(35 \%)$ cases. Eleven (15\%) patients had jaundice. Needle aspiration was successful in $31(86 \%)$ out of the 36 patients, after single aspiration in 10, two aspirations in 18, and three aspirations in 3 patients. PCD was successful in $35(97 \%)$ patients. The median duration of catheter insertion was 13 days (range 6-34 days, mean 12.8 days). Mean (SD) duration of intravenous antibiotic $(\mathrm{p}=0.04)$ and duration to clinical relief $(\mathrm{p}=0.02)$ were shorter in the PCD group (Table 2).

The success rates in the PNA and PCD groups were $86 \%$ and $97 \%$, respectively ( $\mathrm{p}=0.2$ ) (Table 2). PCD failed in one case, who had diabetes and multiple pyogenic liver abscesses involving the right liver lobe. He had rupture of abscess during treatment leading to peritonitis and sepsis and underwent exploration with curettage of abscess cavity along with peritoneal lavage and placement of abdominal drains. He died of persistent sepsis and multi-organ failure. PNA failed in five patients. Two patients had a single amoebic abscess; the other three had multiple pyogenic abscesses in the right lobe of liver. Four of the five failures of PNA group were treated by successful open surgical drainage while one underwent successful laparoscopic surgical drainage. One patient in the aspiration group developed subcapsular hematoma, without hemodynamic 
compromise. In the PCD group, one patient developed rupture of abscess and died; another patient developed transient bile leakage after removal of catheter.

We did not wait for complete radiologic resolution of abscess cavity before discharge; the average size of abscess at the time of discharge was $3.8 \mathrm{~cm}$. The mean (SD) size of abscess at discharge was $3.0 \mathrm{~cm}$ in PCD vs. $4.2 \mathrm{~cm}$ in PNA group $(\mathrm{p}=0.88)$. At 6 months of follow- up, more number of patients treated successfully by PCD $(29 / 35,83 \%)$ had complete sonographic resolution of abscess cavities, compared to patients treated successfully by PNA $(14 / 31,45 \%)(\mathrm{p}=0.02)$. There were four recurrences in aspiration group and two in PCD group at 6 months of follow-up.

\section{Discussion}

Liver abscesses, both amebic and pyogenic, continue to be an important cause of morbidity and mortality in the tropical countries. ${ }^{1}$ Patients usually present late when the liver abscess attains a large size. Percutaneous drainage (either needle aspiration or catheter drainage) with systemic antibiotics has become the preferred treatment for the management of pyogenic liver abscesses. ${ }^{3,5,6,10-13,15,18}$.

Table 2 Treatment response

\begin{tabular}{|l|c|c|c|}
\hline Criteria for response & $\begin{array}{l}\text { Percutaneous needle } \\
\text { aspiration }\end{array}$ & $\begin{array}{l}\text { Percutaneous } \\
\text { catheter drainage }\end{array}$ & p-value \\
\hline Duration of antibiotics(days) & $15.5(1.1)$ & $10.9(2.7)$ & 0.04 \\
\hline Clinical relief attained(days) & $10.2(2.0)$ & $8.1(2.7)$ & 0.02 \\
\hline Duration of hospital stay(days) & $22.2(2.0)$ & $20.3(2.4)$ & 0.08 \\
\hline Success rate & $31 / 36(86 \%)$ & $35 / 36(97 \%)$ & 0.20 \\
\hline
\end{tabular}

Values are as mean (SD)

Table 1 Patient characteristics, blood investigations and abscess characteristics

\begin{tabular}{|l|c|c|}
\hline & $\begin{array}{l}\text { Percutaneous needle aspiration } \\
(\mathrm{n}=36)\end{array}$ & Percutaneous catheter drainage (n=36) \\
\hline Age(yrs)(Median[range]) & $42(16-88)$ & $40(3-72)$ \\
\hline Male: Female & $25: 11$ & $28: 8$ \\
\hline & & 3 \\
\hline Co-morbidity (n) & 5 & 11 \\
\hline Diabetes & 12 & 7 \\
\hline Biliary stones & 4 & 2 \\
\hline Cholangitis & 4 & 1 \\
\hline Previous GI surgery & 0 & \\
\hline Colitis & & 27 \\
\hline & & 10 \\
\hline Clinical features (n) & 29 & 34 \\
\hline Fever & 8 & 22 \\
\hline Rigors, chills & 33 & 3 \\
\hline Right hypochondriac pain & 32 & 14 \\
\hline Anorexia/malaise & 8 & \\
\hline Jaundice & 12 & $9.5(6.6-12.8)$ \\
\hline Nausea/vomiting & & $14.3(3.3-23.4)$ \\
\hline & & $1.65(0.9-2.4)$ \\
\hline Investigations (median & $28: 1: 6$ & $2(0.6-2.5)$ \\
[range]) & $22: 14$ & $47(18-72)$ \\
\hline Hemoglobin (g \%) & & $47(22-69)$ \\
\hline Total leukocyte count(/L) & $13.6(3.8-22.2)$ & $194.5(55-260)$ \\
\hline PT-INR & $1.9(1.0-2.5)$ & 5.5 \\
\hline Bilirubin (mg \%) & $1.8(0.6-2.4)$ & $31: 5$ \\
\hline AST(IU/L) & $45(23-68)$ & $26: 10$ \\
\hline ALT(IU/L) & $51(27-74)$ & \\
\hline Serum ALP & $188(72-266)$ & \\
\hline Total protein (g \%) & 5.75 & \\
\hline Abscess(n)(single:multiple) & $28: 8$ & \\
\hline Location(Right:left:both) & & \\
\hline Nature(Amebic: pyogenic) & & \\
\hline
\end{tabular}

$15 \%$ of these may be refractory to medical therapy, ${ }^{1}$ while $20 \%$ may be complicated by secondary bacterial infection ${ }^{19}$ Such amebic abscesses and those involving left lobe, or those with impending rupture also need to be drained. ${ }^{20}$ Surgical drainage is now used only in cases which fail to respond to percutaneous drainage $^{.3-6}$ Although, PCD is a preferred method most widely used to drain liver abscesses, ${ }^{3,8,21}$ recent studies have shown PNA to be simpler, less costly, and equally effective. ${ }^{8,12,14}$ 
Usually needle aspiration is preferred for smaller abscesses and catheter drainage is done in larger ones. But no clear cut guidelines have been laid. Three previous prospective randomized studies ${ }^{5-7}$ have compared PNA with PCD. All these studies included abscesses of all sizes. Yu et $a l^{6}$ included only pyogenic abscesses and showed no significant difference between the two techniques. Rajak et $a l^{5}$ found that catheter drainage was better terms of success rate, but they limited the number of aspirations to two which may be a reason for lower success rate of percutaneous aspiration. Zerem and $\mathrm{Hadzic}^{7}$ included 60 patients of PLA only, and found PCD to be more efficacious. They recommended PNA for simple abscesses of $5 \mathrm{~cm}$ or smaller in size. We compared these two treatment options, exclusively in liver abscesses $5 \mathrm{~cm}$ or larger. Few reports suggest that the initial size of the abscess cavity does not affect the final outcome, ${ }^{14,22}$ while Rajak et al $l^{5}$ believed that large abscesses are more difficult to evacuate completely in a single attempt.

This may be the reason, why many centers prefer PNA for abscesses $<5 \mathrm{~cm},{ }^{22}$ and PCD for larger abscesses. Both these techniques have certain disadvantages. Multiple attempts of PNA needed for large abscesses may be uncomfortable and perceived as more traumatic by patients. Also during the period between two aspirations pus may get re-accumulated. For smaller abscesses, daily production of pus may be small, but a larger abscess cavity may produce larger quantity of pus, which needs to be drained continuously. PCD has this obvious advantage over PNA, which may have accounted for quicker clinical recovery, lesser duration of parenteral antibiotics and lesser failure rate among patients treated with PCD. On the other hand, placing a catheter needs more expertise followed by nursing care. Thick pus which cannot be drained percutaneously has been reported as the reasons for failure of PCD. We overcame this problem by using larger bore $14 \mathrm{~F}$ catheters for PCD. At 6 months follow up, complete resolution of abscess cavity on USG occurred in two-thirds of 66 patients; 29 of them belonged to the PCD group $(\mathrm{p}=0.02)$. Singh and Kashyap ${ }^{23}$ also reported faster and more complete resolution of abscess cavities after PCD, while Rajak et $a l^{5}$ found that the time needed for total resolution is similar after PCD and PNA. Thus PCD and PNA are equally effective in the management of large liver abscesses. PCD appears to be better in terms of duration to attain clinical relief, duration of intravenous antibiotics needed, and complete resolution of abscess cavity.

\section{References}

[1]. Sharma MP, Ahuja V. Management of amebic and pyogenic liver abscess. Indian J Gastroenterol 2001;20(Suppl 1):C33-6.

[2]. Thompson JE Jr, Forlenza S, Verma R. Amebic liver abscess: a therapeutic approach. Rev Infect Dis 1985;7:171-9.

[3]. Seeto RK, Rockey DC. Pyogenic liver abscess: changes in etiology, management and outcome. Medicine (Baltimore) 1996;75:99113.

[4]. Wong KP. Percutaneous drainage of pyogenic liver abscess. World J Surg 1990;14:492-7.

[5]. Rajak CL, Gupta S, Jain S, Chawla Y, Gulati M, Suri S. Percutaneous treatment of liver abscesses: needle aspiration versus catheter drainage. Am J Roentgenol 1998;170:1035-9.

[6]. Yu SC, Ho SS, Lau WY, et al. Treatment of pyogenic liver abscess: prospective randomized comparison of catheter drainage and needle aspiration. Hepatology 2004;39;932-8.

[7]. Zerem E, Hadzic A. Sonographically guided percutaneous catheter drainage versus needle aspiration in the management of pyogenic liver abscess. Am J Roentgenol 2007;189: W138-42.

[8]. Saraswat VA, Agarwal DK, Baijal SS, et al. Percutaneous catheter drainage of amoebic liver abscesses. Clin Radiol 1992;45:187-9.

[9]. VanSonnenberg E, D'Agostino HB, Casola G, Halasz NA, Sanchez RB, Goodacre BW. Percutaneous abscess drainage: current concepts. Radiology 1991;181:617-26.

[10]. Lambiase RE, Deyoe L, Cronan JJ, Dorfman GS. Percutaneous drainage of 335 liver abscesses: results of primary drainage with 1 year follow up. Radiology 1992;184:167-79.

[11]. Juul N, Sztuk FJ, Torp-Pedersen S, Burcharth F. Ultrasonically guided percutaneous treatment of liver abscesses. Acta Radiol 1990;31:275-79.

[12]. Baek SY, Lee MG, Cho KS, Lee SC, Sung KB, Auh YH.n Therapeutic percutaneous aspiration of hepatic abscesses: effectiveness in 25 patients. Am J Roentgenol 1993;160:799-802.

[13]. Ch Yu S, Hg Lo R, Kan PS, Metreweli C. Pyogenic liver abscess: treatment with needle aspiration. Clin Radiol 1997;52:912-6.

[14]. Giorgio A, Tarantino L, Mariniello N, et al. Pyogenic liver abscesses: 13 years of experience in percutaneous needle aspiration with US guidance. Radiology 1995; 195:122-4.

[15]. Mohsen AH, Green ST, Read RC, McKendrick MW. Liver abscess in adults:ten years experience in a UK centre. Q Med 2002;95:797-802.

[16]. Pitt HA. Surgical management of hepatic abscesses. World J Surg 1990;14:141-52.

[17]. Wong WM, Wong BC, Hui CK, et al. Pyogenic liver abscess: retrospective analysis of 80 cases over a 10-year period. J Gastroenterol Hepatol 2002;17:1001-7.92 Singh, et al. 1 Springer Indian J Gastroenterol 2009(May-June):28(3):88-92

[18]. Chou FF, Sheen-Chen SM, Chen YS, Chen MC, Chen CL. Single and multiple pyogenic liver abscesses: clinical course, etiology, and results of treatment. World J Surg 1997;21:384-9.

[19]. Sherlock S, Dooley Y. Disease of the liver and biliary system. 9th ed. Oxford: Blackwell, 1993:pp. 471-502.

[20]. Sharma MP, Rai RR, Acharya SK, Ray JC, Tandon BN. Needle aspiration of amoebic liver abscess. BMJ 1989; 299:1308-9.

[21]. 21.HashimotoL,Hermann R,Grundfest-Broniatowski S.Pyogenic hepatic abscess: results of current management. Am Surg 1995;61:407-11.

[22]. Stain SC, Yellin AE, Donovan AJ, Brien HW. Pyogenic liver abscess: modem treatment. Arch Surg 1991;126: 991-6.

[23]. Singh JP, Kashyap A. A comparative evaluation of percutaneous

[24]. catheter drainage for resistant amebic liver abscesses. 